poetischen ausdrucken und umschreibungen operiert, zu rechnen ist.

Ich lasse hier noch eine neue erklärung von Hárr, dem beinamen órins, folgen:

Die regelrechte nord. entsprechung von got. haihs $\mu$ ovó$\varphi \vartheta \alpha \lambda \mu \rho \sigma_{\text {Marc. }} 9,47$ mlisste hárr lauten, so wie dem got. faihs an. fárr entspricht. Der beiname Óðins könnte also ursprünglich 'der einäugige' bedeutet haben, so wie bei Saxo Othinus als der altero oculo orbus erseheint, s. 0. 8. 74, vgl. auch den Svipdagr blindi, oben s. 93, den Gestiblindus, Gestr hinn blindi, oben s. 86. Die bedeutung 'der hohe' hätte das wort erst später erbalten.

WIEN.

FERD. DETTER.

\title{
THEOTISCUS. DEUTSCH.
}

Jacob Grimm hat in der Grammatik 1 3 , 13 ff. die geschichte dieses wortes skizziert; neue daten weiss ich keine anzugeben. Demnach kommt es als bezeichnung der sprache zuerst 788 in den Lorscher annalen vor (Mon. Germ. 1, 172)1), also in einer lateinischen aufzeichnung fränkischen ursprungs, von da an als lateinisches wort in einer reihe von quellen besonders wider fränkischen ursprungs. In deutschem context erscheint es erst viel spăter. Otfrid hat es als deutsches wort nicht (s. u.); die von Graff, Sprachschatz 5, 130 gegebene stelle Germania thiudisca liudi in den Strassburger altsächsischen Isidorglossen würde es weiter hinauf rutcken, denn Graff setzt die hs. ins 8., 9. jh.; aber ich habe auch mit Ed. Sievers' freundlicher hilfe uber das wirkliche alter der verbrannten hs. nichts finden können. Im

1) Die einzelnen abschnitte dieser annalen sind in der tat mit den ereignissen ziemlich gleichzeitig, was Grimm bezweifelte. Die von ihm vorangestellte stelle von 813 ist also nicht die älteste. Den beweis, den man aus dem Daniel-scholion zu Aen. 7, 741: cateiae lingua Theotisca hastae dicuntur, fiir ein höheres alter des wortes entnehmen könnte, hat Franz Cramer, Archiv für lat. lexikogr. 5, $141 \mathrm{f}$., glieklich entkräftet. Für einen westgotischen ursprung des scholions, wie ihn H. Georgii, Die antike Aeneiskritik 21, annimmt, scheint mir gar nichts za sprechen. 
ubrigen ist das wort als deutsches vor dem 10. jh. nicht nachzuweisen (Grimm a. a. o. 15).

Grimm hat aus diesem eigentumlichen verhalten, dass ein wort deutscher etymologie ein jahrhundert lang nur in lateinischen quellen erscheint, keinen weiteren schluss gezogen. $\mathrm{Er}$ war dem richtigen nahe; aber wie öfters liess die scheu, fremden einfluss anzunehmen, das streben, alles als echte und alte volkstradition zu erweisen, ihn nicht ganz dazu gelangen. $\mathrm{Er}$ sagt a. a. 0. 14: 'wer nun aus diesen stellen folgern wollte, erst im neunten $j$., seit Carl der grosse die deutschen stämme stärker vereinte, sei die allgemeine benennung entsprungen, würde fehlen'. Und doch ist das die einzige mögliche ansicht. Es begreift sich ohne weiteren beweis: wenn ein gemeinsames wort für die sprachen aller continentalen Westgermanen erst von der zeit an nachzuweisen ist, wo diese völker zum ersten male in einem reiche vereinigt waren - und das war seit der unterwerfung der Sachsen der fall -, so ist es mindestens für den begriff, den es bezeichnet, auch erst von da an gebraucht worden. Es war vorher kein bedurfnis einer solchen gemeinbezeichnung vorhanden. Alcuin hat einmal 786 das schon von den alten im weiteren $\sin n=$ germanisch gebrauchte teutonicus fur die sprache seiner angelsächsischen landsleute gebraucht (Jaffé, Bibl. rer. germ. 6,160 f.); dieses ist aber erst im 10. jh. allgemeiner geworden (s. Dummler, Otto d. gr. $562 \mathrm{ff}$ ); und dass man ein feststehendes wort für deutsche sprache fruber in der tat nicht hatte, geht aus den stellen des 6.-8. jh. hervor, welche Grimm a. a. $0.12 \mathrm{f}$. citiert hat, wo ausweichende ausdrücke wie eorum lingua, sermo barbaricus, lingua propria, vulgo u. ä. gebraucht sind oder aber der name des einzelnen volksstammes.

Aber auch um 870 kann das wort als deutsches wort noch nicht geläufig gewesen sein; das beweist Otfrid zur gentige, der (s. Kelle 1, 13 ff.) in seinem lateinischen text sieben mal theotiscus von der sprache gebraucht, im deutschen nie, dagegen an acht stellen des letztern frenkisg setzt. Er musste das wort gebraucht haben, wenn er es gekannt hätte.

Ich schliesse daraus, dass das wort, obwol eine deutsche sprachbildung, seinen ursprung doch nicht im volkstimlichen gebrauch, sondern nur in gelehrten kreisen haben kann. Es 
ist, aus dem beduirfnis einer generellen bezeichnung heraus, in denjenigen kreisen aufgekommen, welche das dringendste bedurfnis einer solchen empfinden mussten: in den officiellen und literarischen hofkreisen; und es ist wol kaum ein zufall, dass es zuerst in den officiösen Lorscher annalen, dann in synodalbeschltissen, bei historikern u. s. w. vorkommt. Die möglichkeit ist sogar nicht zu leugnen, dass das wort künstlich gebildet ist. Denn als gemeingermanisch ist es nicht zu erweisen. Wulfilas piudiskô für $\hat{\varepsilon} \vartheta v \iota x \tilde{c}_{\varsigma}$ ist gewiss von ihm selbst gebildet, weil er an der stelle (Gal. 2,14) nicht ohne eine adverbialbildung auskam; hätte er das adjectiv im gotischen vorrätig gefunden, so hätte er nicht oi ç่vixoì zweimal (Mt. 5, 46. 6, 7) mit pai piudô ubersetzt. Ags. péodisc ist substantiv, die skandinavischen bezeichnungen sind aus dem deutschen entlehnt, und githiuti bei Otfried, githiudo im Heliand gehören nicht hierher. Ich will es jedoch nur als möglichkeit hinstellen, dass die wortbildung theotiscus als solche gelehrten ursprungs sei: die verwendung für germanicus ist sicher dieses ursprungs. Wenn aber ein wort, das ein jahrhundert lang im officiellen gebrauch war, dann in die volkssprache tubergegangen ist, so darf das nicht wunder nehmen.

TÜBINGEN, 12. mai 1893.

HERMANN FISCHER.

\section{ZUM REINHART FUCHS.}

Dass der dichter des Reinhart Fuchs in dem olbentenabenteuer eine anspielung auf zoitereignisse gemacht hat, ist allgemein anerkannt, und man hat sich eifrig bemuht, in die beziehungen dieser erzählung licht zu bringen. Allein bisher sind die bestrebungen nicht von erfolg begleitet gewesen: tiber vermutungen allgemeiner art sind, von allteren autoren abgesehen, weder Reissen berger (Reinh. Fuchs s. 16 f.) noch Martin (Observations sur le roman de Renart s. 108 f.) hinausgekommen. Doch haben sie die grundlinien des verständnisses richtig gezogen: es ist sehr einleuchtend, wenn Martin annimmt, dass es sich bei der ausgesprochen kaiserlichen gesinnung der reichsabtei Erstein um den versuch einer vergewaltigung seitens der päpstlichen partei handele. Ueber die dabei in betracht kommenden factoren sollen die nachfolgenden zeilen eine ver- 